\title{
Commentary
}

\section{Large Urban Developments and the Future of Cities: The Case of Neighborhoods}

\author{
Emily Talen \\ Social Sciences Division, University of Chicago, Chicago, IL 60637, USA; E-Mail: talen@uchicago.edu
}

Submitted: 6 November 2019 | Accepted: 6 November 2019 | Published: 21 November 2019

\begin{abstract}
The production of neighborhoods on a large or mass scale has not been successful. Procuring the neighborhood ideal requires an attention to detail that few large corporations or government agencies seem capable of instituting. Yet planned neighborhoods have definite pluses: institutionalized leadership, clearly defined social and spatial boundaries, and a sense of control. What is needed is an approach that combines the best of both worlds-a dose of planning, with plenty of flexibility and local empowerment.
\end{abstract}

\section{Keywords}

incremental urban development; neighborhoods; top-down planning

Issue

This commentary is part of the issue "Large Urban Developments and the Future of Cities" edited by Efrat Eizenberg (Technion-Israel Institute of Technology, Israel).

(C) 2019 by the author; licensee Cogitatio (Lisbon, Portugal). This article is licensed under a Creative Commons Attribution 4.0 International License (CC BY).

Large, corporate urban development deserves deep and careful critique. We can look at the experience of largescale neighborhood development to better understand the intrinsic problems such developments engender. Planning for neighborhoods has always required certain nuances and sensibilities-nuances that large-scale master planning has rarely been able to muster. When mass produced or produced on a large scale, neighborhoods tend to be reduced to single-use, monolithic, and mostly suburban developments. In particular, the principles of mixed housing type oriented around communal space are usually dropped.

Successful emulation of the complete neighborhood ideal requires an attention to detail that few large corporations or government agencies are capable of instituting. In the US, the federal government ordered that housing be constructed in the form of neighborhoods starting in the 1940s, but the models were severely diluted. The government's hugely impactful Successful Subdivisions: Planned as Neighborhoods for Profitable Investment and Appeal to Home Owners bulletin equated "subdivision" with "neighborhood" and directed subdividers to keep lots uniform in size and to "segregate uses," because, despite the benefits for pedestrians, "short blocks are not economical" (Federal Housing Administration of the United States [FHAUS], 1940, p. v). Listed as causes of depreciated real estate values and the "break down of neighborhood character" were business uses "invading residential areas" and "mixtures of apartments and detached dwellings" (FHAUS, 1940, p. v). There was an attempt at nuance-stores were to be "conveniently located within walking distance" and shopping centers were to be "restricted in extent" (FHAUS, 1940, p. v), but these subtle restrictions were not maintained by developers, and massive shopping centers surrounded by parking lots became the norm.

The whole proposition of planning by neighborhood became suspect. It was a rejection of the planned neighborhood-of thinking about how neighborhoods ought to be rather than simply affirming their existing form. Critics of the planned neighborhood assumed that its repeated failures in practice were endemic to the model-and especially, endemic to mass produced, large-scale development, about which there seemed little alternative.

What are the alternatives to large-scale, all-at-once, top down neighborhood development? What is needed is an approach that combines the best of both worlds-a 
dose of planning, with plenty of flexibility. Planned neighborhoods have definite pluses: institutionalized leadership, clearly defined social and spatial boundaries, and a sense of control. Unplanned neighborhoods are likely to lack these advantages.

Unfortunately, nuancing the plan versus process balance that neighborhoods might benefit from has rarely happened. Neighborhood plans have instead been mostly wholesale, all-at-once, expert driven formulations. Missing has been the transfer of the neighborhood ideal in incremental terms-redeveloping existing places one block at a time. Urban planners never developed a language or methodology that could implement the ideals of neighborhood as a physical and social construct in a way that was not top-down-not about blueprints, but not limited to process, either-plan and process combined.

The complexities of this balancing act come into view in the attempt to rely on incremental change as a way of improving neighborhoods. If there is no understanding of how incremental achievement leads to the gradual building up of something whole, with no ties to neighborhood, small improvements may seem like piecemeal shots in the dark, benefitting one landlord, one property owner, one gentrifier at a time. Would these catalytic efforts be that much more effective if they were contextualized within an identified neighborhood? A top-down plan is not necessarily the answer, but a clearer connection to a defined neighborhood may help broaden and deepen these efforts.

Plan versus process reveals the tension between collective input that requires planning protocols, and the desire for an agile response in the form of pop-up shops, bench bombing, and painted crosswalks. There is a need for spontaneity and there is a need for representation that is fair and democratic. Perhaps, at least, an explicit understanding of neighborhood and its attendant notions of collective enterprise, responsibility, and ownership could help resolve the two extremes of centralized planning versus DIY intervention.

There is always the danger that small-scale efforts combined with a strong sense of neighborhood will be over-played, resulting in an escalation of housing prices and eventual displacement. Neighborhood improvement without disruption and displacement is based on the idea that improvements must be defined by residents themselves.

Narratives surrounding climate change, sustainability, and resiliency could potentially help resolve the dichotomy that pits bottom-up authenticity against neighborhood plans and planning. Neighborhood-scale governance and control is important for environmentalism because neighborhood scale is used as a basis of sustainable practices-e.g., water conservation, groundwater recharge, recycling, energy efficiency, and food production. Individual actions matter too, but many sustainability and resilience goals require local coordination, where the scale of operations is at the neighborhood level.

The processes of neighborhood-tactical, empowering, bottom-up, environmentally-based-requires a defined-to some extent, planned-neighborhood. But it need not be large-scale and corporate. Individual possibility can be maximized, with minimal limits on opportunity and movement, within the context of bounded urban space. The goal of planners should be to derive an individualized urban experience composed of varying and unbounded social worlds, while at the same time recognizing that the form and design of the neighborhoodif it adheres to certain principles-promotes neighborhood identity and, potentially, civic identity and spirit.

This is the balance between process and plan that has to be found, a sense of neighborhood versus the freedom to engage, small-scale intervention that adds up, neighborhood identity that does not impose too much control and too much order.

\section{Conflict of Interests}

The author declares no conflict of interests.

\section{References}

Federal Housing Administration of the United States. (1940). Successful subdivisions: Planned as neighborhoods for profitable investment and appeal to home owners (Land Planning Bulletin No. 1). Washington, DC: Federal Housing Administration of the United States.

\section{About the Author}

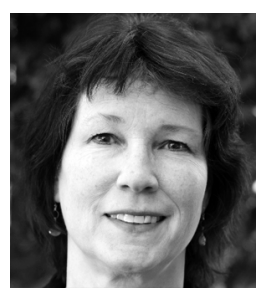

Emily Talen is Professor of Urbanism at the University of Chicago, where she teaches urban design and directs the Urbanism Lab (http://urbanism.uchicago.edu). She holds a PhD in urban geography from the University of California, Santa Barbara and a master's in city planning from Ohio State. She is a Fellow of the American Institute of Certified Planners, and the recipient of a Guggenheim Fellowship. Talen has written extensively on the topics of urban design, New Urbanism, and social equity. Her books include New Urbanism and American Planning, Design for Diversity, Urban Design Reclaimed, City Rules and Neighborhood. She is co-editor of the Journal of Urbanism. 